FORMATION Formation emploi

Revue française de sciences sociales

146 | Avril-Juin 2019

L'apprentissage en Allemagne face à ses défis

\title{
Répondre aux besoins des diplômés de l'enseignement professionnel allemand : vers une perméabilité institutionnelle?
}

Supporting needs of vocationally qualified students - changes towards institutional permeability in Germany?

Unterstützung von Bedürfnissen beruflich qualifizierter Studierender in

Deutschland: Entwicklungen in Richtung institutioneller Durchlässigkeit?

Responder a las necesidades de los graduados de la enseñanza técnica alemana:

¿hacia una permeabilidad institucional?

\section{Nadine Bernhard}

\section{OpenEdition}

Journals

Édition électronique

URL : http://journals.openedition.org/formationemploi/7255

DOI : 10.4000/formationemploi.7255

ISSN : 2107-0946

Éditeur

La Documentation française

Édition imprimée

Date de publication : 26 juin 2019

Pagination : 129-147

ISSN : 0759-6340

Référence électronique

Nadine Bernhard, «Répondre aux besoins des diplômés de l'enseignement professionnel allemand vers une perméabilité institutionnelle?», Formation emploi [En ligne], 146 | Avril-Juin 2019, mis en ligne le 01 janvier 2021, consulté le 07 janvier 2021. URL : http://journals.openedition.org/formationemploi/ 7255 ; DOl : https://doi.org/10.4000/formationemploi.7255 


\title{
Répondre aux besoins des diplômés de l'enseignement professionnel allemand : vers une perméabilité institutionnelle?
}

\author{
NADINE BERNHARD \\ Post-doctorante à l'université Humboldt de Berlin, \\ Centre pour l'éducation internationale et comparée
}

Résumé

Répondre aux besoins des diplômés de l'enseignement professionnel allemand: vers une perméabilité institutionnelle?

Les structures d'appui répondant aux besoins hétérogènes d'un nombre croissant d'étudiants de l'enseignement supérieur, diplômés de l'enseignement professionnel, peuvent aider ces derniers à réussir leurs études et ainsi favoriser la perméabilité entre l'enseignement et la formation professionnels (EFP) et l'enseignement supérieur. Cet article analyse l'évolution de ce type de structures, en Allemagne, en mobilisant une analyse institutionnelle multidimensionnelle. En Allemagne, la reconnaissance croissante des étudiants titulaires de diplômes professionnels comme groupe d'étudiants légitime rend de plus en plus nécessaire la réponse à leurs besoins. Toutefois, les hiérarchies entre les étudiants " traditionnels » et les étudiants diplômés de l'enseignement professionnel tendent à se reproduire.

Mots clés : enseignement supérieur, enseignement technique-professionnel, système éducatif, cheminement scolaire, accompagnement scolaire, Allemagne

Abstract

Supporting needs of vocationally qualified students - changes towards institutional permeability in Germany?

Structures that respond to the diverse needs of the increasing number of vocationally qualified students in higher education (HE) can facilitate their study success and, thereby promote permeability between vocational education and training (VET) and HE. This article analyses the evolution of this type of support structures in Germany with the help of differentiated institutional analysis. With the increasing recognition of the vocationally qualified as a legitimate group of students in Germany, dealing with their needs is becoming more critical. However, hierarchies between " traditionals " and vocationally qualified students are reproduced.

Keywords: higher education, technical \& vocational education, education system, school paths, school support, Germany

Journal of Economic Literature: I 21 ; I 23

Traduction : Auteure. 


\section{Formation professionnelle et perméabilité : limites de l'égalité des chances}

Le système allemand d'enseignement et de formation professionnels (EFP) jouit d'une excellente réputation pour diverses raisons, tenant par exemple au faible taux de chômage des jeunes, à l'accès relativement aisé au marché du travail, et aux possibilités de formation continue au sein du système de formation professionnelle supérieur, en dehors des universités. En outre, le système allemand de formation professionnelle permet d'acquérir des compétences de haut niveau grâce à un système collectif de formation professionnelle dans une économie de marché coordonnée. Ce système conduit à une production diversifiée de qualité, considérée comme un facteur crucial pour la compétitivité économique de l'Allemagne (Busemeyer \& Trampusch, 2012 ; Euler, 2013).

Un aspect souvent inobservé est le problème que Baethge (2006) nomme le «schisme éducatif » : le système d'EFP et le système d'enseignement général secondaire et supérieur se sont développés en grande partie séparément, ce qui rend difficiles les transitions de l'un à l'autre. Dans un tel système d'éducation non perméable, les personnes qualifiées dans le cadre d'une formation professionnelle sont par conséquent exclues des possibilités de formation continue dans le secteur académique qui, en moyenne, offre encore les meilleures perspectives individuelles de carrière. Contrairement à d'autres pays, comme la France, où le baccalauréat technique et professionnel permet d'accéder au système d'enseignement supérieur par la voie professionnelle, ces possibilités de transition n'ont toujours pas été institutionnalisées en Allemagne, à quelques exceptions près (Powell \& al., 2012).

La perméabilité institutionnelle du système éducatif est une condition préalable à la mobilité éducative et sociale. Elle implique l'absence d'impasse éducative et la mise en place de voies de transition souples et pleinement opérationnelles. Par conséquent, la question de la perméabilité institutionnelle est essentielle dès lors que des systèmes éducatifs stratifiés, sans liens entre les différents secteurs de l'éducation, entravent très probablement l'égalité d'accès à l'éducation et à l'emploi.

Dans le même temps, dans cet article, la perméabilité institutionnelle n'est pas envisagée de façon stricte : elle dépasse en effet la simple possibilité de passer d'un système de formation à l'autre. D'un point de vue analytique, le concept de perméabilité institutionnelle peut être analysé à l'aune de quatre dimensions (Bernhard, 2017a) : l'accès à l'éducation, la reconnaissance et la validation des acquis, les liens organisationnels et les structures de soutien institutionnel, dont le rôle est de répondre aux besoins hétérogènes des apprenants (voir partie 2).

En Allemagne, l'accès à l'enseignement supérieur par la formation professionnelle a été assoupli, dans une certaine mesure, ces dernières années (Ulbricht 2012). Ainsi, un plus grand nombre de personnes diplômées de l'enseignement professionnel cherchent à accéder au système d'enseignement supérieur (Wolter \& al., 2017, pp. 14-15). Dans 
ce contexte d'évolution des possibilités d'accès à l'éducation, il est également nécessaire d'analyser dans quelle mesure cela engendre aussi des changements institutionnels au sein des structures d'appui, dont le rôle est de prendre en compte les différents besoins des diplômés de l'enseignement professionnel non titulaires du baccalauréat de l'enseignement supérieur. Face à ce nouveau groupe d'étudiants, comment les organisations de l'enseignement supérieur doivent-elles réagir et s'adapter, tant dans leur structure que dans leurs pratiques ? Et comment ces structures ont-elles évolué, en Allemagne, entre 1990 et 2012, avec l'ouverture de l'accès à l'enseignement ? En analysant les structures d'appui institutionnelles, comme les services d'information et de conseil, les dispositifs permettant d'étudier à temps partiel ou encore des concepts pédagogiques adaptés, l'article vise à s'interroger sur la place des étudiants issus de l'enseignement professionnel au sein du système d'enseignement supérieur : sont-ils considérés comme des étudiants légitimes, dont les besoins doivent être reconnus et qui doivent être accompagnés dans la réussite de leurs études, ou bien sont-ils seulement autorisés à accéder, mais aussi à s'adapter, à ce nouvel environnement ? L'article montre également comment ce groupe d'étudiants se construit progressivement en se démarquant des étudiants " traditionnels ».

Pour répondre à ces problématiques de recherche, la conceptualisation de la perméabilité institutionnelle est, dans un premier temps, esquissée, avant d'être suivie d'une illustration des problèmes de perméabilité en Allemagne. Ensuite, l'approche théorique institutionnelle et discursive qui guide ce travail, ainsi que l'approche méthodologique utilisée sont discutées. L'analyse de ces structures est effectuée selon une compréhension large des institutions (Scott, 2008). Elle examine les changements réglementaires en Allemagne, mais se concentre également sur la dimension institutionnelle normative et culturo-cognitive. À cet effet, elle analyse les discours de politique éducative, au sein desquels les normes et les réflexions des acteurs sociaux sur le thème de la perméabilité entre l'EFP et l'enseignement supérieur, en général, et les structures de soutien, en particulier, peuvent apparaître. Enfin, nous présentons les développements réglementaires et discursifs en Allemagne. L'article fournit un bref aperçu de l'évolution de la perméabilité institutionnelle dans ce pays, en mettant particulièrement l'accent sur la perméabilité des structures de soutien.

\section{Perméabilité : conceptualisation et description de la problématique}

La perméabilité est un concept souvent utilisé, mais rarement défini dans la littérature éducative (voir cependant Wolter \& al., 2014). Au sein de ce concept, il est possible de distinguer la perméabilité sociale au sens de la mobilité sociale et la perméabilité institutionnelle. Cette dernière met l'accent sur la conception institutionnelle du système éducatif et invite à se demander dans quelle mesure elle permet aux apprenants de passer d'un domaine ou d'un cours à un autre. 
La perméabilité institutionnelle peut être horizontale, c'est-à-dire qu'elle peut concerner le passage d'un programme éducatif à un autre relevant d'un même niveau d'éducation, ou verticale, c'est-à-dire qu'elle peut concerner la transition vers un programme éducatif relevant d'un niveau supérieur. Cette partie décrit la conception de la perméabilité institutionnelle, y compris de la perméabilité verticale et horizontale, puis présente les structures de perméabilité institutionnelle en Allemagne.

\subsection{Accès, reconnaissance, liens organisationnels et soutien pour des besoins hétérogènes}

La conception analytique de la perméabilité a été élaborée principalement sur la base d'une analyse systématique de la littérature existante en matière de perméabilité entre l'enseignement supérieur et la formation professionnelle. Elle repose sur les questions générales suivantes : quels sujets et structures institutionnelles, abordés dans la littérature, permettent et facilitent les transitions des apprenants et la réussite de l'apprentissage entre les domaines éducatifs ? Comment réduire la fracture institutionnelle entre l'enseignement supérieur et l'EFP ? Quatre dimensions différentes ont pu être identifiées (Bernhard, 2017a) : l'accès à l'éducation, la reconnaissance et la validation, les liens organisationnels et les structures de soutien institutionnel.

La première dimension est l'accès à l'éducation. Il s'agit de la dimension à laquelle la plupart des gens pensent lorsqu'ils parlent de perméabilité. Cet accès peut être illimité et permettre ainsi un maximum de perméabilité. Dans ce cas, les apprenants n’ont pas à remplir de conditions préalables. Il en va ainsi des cours en ligne ouverts à tous et gratuits (MOOC). Par ailleurs, l'accès peut provenir de droits résultant de l'obtention d'un certificat. Par exemple, en Allemagne, les titulaires du certificat d'entrée dans l'enseignement supérieur ont le droit d'accéder à l'enseignement supérieur. Enfin, l'organisation d'enseignement particulier peut définir elle-même ses propres modalités d'accès, par exemple en conditionnant l'entrée à cet établissement à la réussite de tests d'aptitude, ce qui peut accroître la perméabilité : c'est le cas notamment lorsqu'il importe peu de savoir comment et où les personnes ont acquis les compétences nécessaires à la réussite de ces tests. Il est également possible que l'accès soit conditionné à la fois à l'obtention de certificats d'admissibilité et au respect de modalités d'accès déterminées par l'organisation.

La deuxième dimension, la reconnaissance et la validation des acquis, repose sur l'hypothèse que des parcours d'apprentissage différents peuvent mener à l'acquisition de compétences et de connaissances équivalentes. Il s'agit ici d'examiner si et dans quelle mesure les connaissances et les compétences acquises précédemment correspondent à celles du cursus scolaire cible et peuvent donc remplacer des éléments du programme éducatif.

La troisième dimension est relative aux liens organisationnels entre les différents domaines de l'éducation, l'accent étant mis ici sur le lien entre la formation professionnelle et la formation académique. Cette dimension inclut, d'abord, des liens consécutifs et institu- 
tionnalisés, au sein du parcours de l'étudiant, entre les différents programmes éducatifs, ce que l'on appelle les passerelles en France. Ensuite, différents domaines de l'éducation peuvent également être intégrés au sein d'un cursus ou d'une organisation pédagogique (Bernhard 2017a). Par exemple, l'intégration est possible dans les programmes d'études, la didactique et les lieux d'instruction, ou par la mise en place de doubles qualifications (Young \& al., 1997). Les programmes allemands d'études en alternance (Duale Studiengänge) en sont un exemple : ils se sont développés au début des années 1970 pour pallier la séparation institutionnelle entre l'EFP et l'enseignement supérieur (voir Graf, 2013 ; Baethge \& Wolter, 2015) en reliant, de manière coordonnée et systématique, formation en entreprise et formation dans les établissements d'enseignement supérieur.

La quatrième dimension de la perméabilité, au coeur du présent article, concerne les structures institutionnelles visant à répondre aux besoins hétérogènes des étudiants (Hanft \& al., 2015 ; Wolter \& al., 2014). D’une part, elles offrent un soutien aux apprenants et motivent ces derniers à choisir un cursus approprié. D'autre part, ces structures doivent permettre de garantir une formation réussie dans le cadre du programme éducatif lui-même. Ces structures peuvent prendre plusieurs formes (Bernhard, 2017a) :

- les services d'information et de conseil, avant et après qu'ait été prise la décision de poursuivre des études ;

- les structures de soutien financier, comme les bourses d'études ou d'autres mesures d'aide publique telles que celles prévues par la loi fédérale allemande sur l'aide à la formation (BAföG), qui visent à subventionner les étudiants en fonction du revenu de leurs parents ;

- une adaptation de la façon dont l'apprentissage est organisé pour répondre à l'hétérogénéité des besoins, par exemple par le biais de structures d'études personnalisées permettant de proposer différents rythmes et lieux d'études. De même, l'orientation curriculaire et didactique de l'offre éducative peut être développée afin de faire face aux besoins divers et aux caractéristiques propres des apprenants ;

- une culture d'ouverture au sein des organisations éducatives face à une hétérogénéité accrue des apprenants, cette dernière allant à l'encontre de l'image d'un corps étudiant homogène. Elle vise la reconnaissance de tous les groupes d'étudiants en favorisant l'ouverture d'esprit et la sensibilisation des employés de l'organisation à la problématique de la diversité, par la mise en place de mesures permettant, par exemple, de gérer la diversité.

En résumé, la perméabilité institutionnelle signifie bien davantage que l'octroi de possibilités d'accès et de transition d'un domaine éducatif à un autre. Cette compréhension multidimensionnelle peut être utile pour découvrir les conditions structurelles d'une formation et d'un enseignement réussis. 


\subsection{Une forte division institutionnelle qui engendre des problèmes de perméabilité}

Une fois le concept de perméabilité analysé, comment décrire le système éducatif allemand en matière de perméabilité entre l'enseignement supérieur et la formation professionnelle?

Ce système se caractérise par une forte division institutionnelle entre l'EFP et l'enseignement général, y compris supérieur (Baethge \& Wolter, 2015). Le fameux système allemand de formation professionnelle s'est surtout développé indépendamment de l'enseignement supérieur, ce qui a conduit à un manque de voies d'accès direct à ce dernier pour les diplômés de l'enseignement professionnel. L'accès à l'enseignement supérieur allemand est généralement possible après l'obtention, dans le cadre de l'enseignement général, de l'Abitur (le baccalauréat allemand), c'est-à-dire du certificat d'entrée dans l'enseignement supérieur.

En Allemagne, "la logique institutionnelle générale sous-jacente de la ségrégation dans l'apprentissage " (Powell \& Solga, 2011, p. 54) prend racine dans le système scolaire. Cette division est renforcée dès lors que chaque cursus scolaire, suite à l'obtention du certificat de fin d'études secondaires correspondant, mène à différentes options de formation. Tandis que les cursus s'achevant après le premier cycle de l'enseignement secondaire conduisent traditionnellement à l'EFP, la poursuite du cursus scolaire au lycée, c'est-à-dire au niveau secondaire supérieur, où les élèves reçoivent l'Abitur, conduit principalement à l'enseignement supérieur. L'accès au système d'enseignement supérieur par la voie de la formation professionnelle a été ardu : les diplômés de l'EFP devaient généralement réintégrer le système d'enseignement général et passer leur baccalauréat. D’autres possibilités, comme la réussite de tests d'entrée ou des périodes d'études probatoires, pouvaient être proposées dans le cadre des réglementations existantes prévues par les lois sur l'enseignement supérieur des États fédéraux, ces dernières pouvant fortement diverger (Ulbricht, 2012).

En outre, la ségrégation dans les différents types d'école, puis dans l'enseignement supérieur et la formation professionnelle, engendre simultanément de la ségrégation sociale (Shavit \& Müller, 2000) et se reflète dans la forte sélectivité sociale régnant dans l'accès à l'enseignement supérieur (Pollak \& Reimer, 2010). Ainsi, la question de la perméabilité est particulièrement cruciale dans les systèmes éducatifs pouvant être qualifiés de "très ségrégués", comme celui de l'Allemagne (Kerckhoff, 2001).

Quant à la question de la reconnaissance et de la validation, les compétences acquises dans un système d'enseignement, qu'il s'agisse de l'EFP ou de l'enseignement supérieur, ont rarement été reconnues par l'autre système. En effet, ce n'est que depuis 2002 que 50 \% d'un programme d'études peut être validé dans le cadre d'une recon-

1. Traduction de l'auteure. 
naissance des acquis non académiques. Cependant, il n'existe pas de procédures normalisées de reconnaissance et de validation des compétences (Bernhard, 2017a). Des liens organisationnels, sous forme de programmes d'études en alternance, existent depuis la fin des années 1970 et se sont fortement développés, en particulier au cours des dernières années (Graf, 2013). En outre, le nombre de personnes diplômées de l'enseignement professionnel et non titulaires d'un Abitur étant faible, les structures visant à les soutenir étaient pratiquement inexistantes. La FernUniversität de Hagen, créée en 1974, est la seule université d'enseignement à distance financée par l'État.

Néanmoins, la perméabilité entre l'enseignement supérieur et la formation professionnelle demeure traditionnellement faible au sein du système éducatif allemand.

\section{Analyser le développement institutionnel à l'aune des réglementations et des discours}

L'approche théorique institutionnelle et discursive qui guide ce travail, ainsi que l'approche méthodologique sont décrites ci-après.

En mobilisant la définition globale des institutions de Scott (2008), les changements dans les structures de perméabilité institutionnelle sont analysés en se centrant non seulement sur la dimension régulatrice, mais aussi sur la dimension culturo-cognitive et normative. Ainsi, l'accent est mis sur les politiques et les règlements, mais aussi sur les normes et les idées. Les normes, les valeurs et les standards sont le fondement de la dimension normative mise en œuvre par le mécanisme de l'obligation (morale). Les acteurs agissent alors en fonction de la perception qu'ils ont de leur rôle social et de la connaissance du comportement attendu et classé comme "juste » et "bon " (Scott, 2008). En effet, l'analyse institutionnelle consiste à étudier les normes et standards des organisations d'enseignement supérieur en matière de perméabilité et à identifier les groupes cibles considérés comme légitimes au sein des systèmes d'enseignement professionnel et supérieur.

Enfin, la dimension culturo-cognitive englobe des notions communes de réalité sociale, de systèmes de croyances et de structures de sens qui s'affirment par le mécanisme d'une "chose allant de soi" (Scott, 2008, p. 58). L'analyse porte ensuite sur la manière dont la perméabilité entre la formation professionnelle et l'enseignement supérieur est comprise et légitimée.

Les idées et les normes sur la perméabilité sont analysées à l'aune de la "Wissenssoziologische Diskursanalyse» (WDA)2 (Keller, 2006 ; 2011), dans la construction de la signification de la perméabilité au niveau des pratiques discursives des acteurs

2. ou analyse du discours à l'aune de la sociologie des connaissances. 
sociaux. WDA est un programme de recherche ancré dans la tradition de la sociologie de la connaissance. Il vise à analyser la construction discursive des ordres symboliques (Keller, 2011). À partir des lectures de Foucault, Keller (2006, p. 125), comprend les discours comme des pratiques régulées de production interprétative et de construction de la réalité par lesquelles des structures sociales de la connaissance sont créées. Cette création est liée à une constellation de pouvoirs historiquement située. La répartition du pouvoir et les intérêts des groupes sociaux sont décisifs pour déterminer quelle interprétation concurrente devient hégémonique et se cristallise dans les institutions. Dans les discours, les acteurs sociaux déterminent ainsi les éléments essentiels de la structure de la connaissance d'une société et leur compréhension de la réalité.

Puisque les structures de la connaissance aident les individus à interpréter et structurer le monde et (dé)légitiment les actions politiques et sociales, ainsi que les institutions, les changements les concernant peuvent donc indiquer et conduire à des changements dans ces structures institutionnelles. Cette perspective permet de reconstruire le changement institutionnel, au fur et à mesure que les discussions discursives sur la transformation des définitions sociétales, et donc de certaines parties des déterminations structurelles de l'ordre social, entrent en jeu (Bernhard, 2017a).

\section{Encadré 1 . Approche analytique du discours et analyse documentaire (*)}

Pour retracer les développements institutionnels, l'analyse du discours, selon la WDA (Wissenssoziologische Diskursanalyse)(**) (voir Keller, 2006, 2011), est au cœur de l'article. Mobiliser la WDA revient à faire de l'analyse interprétative. Cela signifie que la recherche discursive relie différents types de données et diverses étapes d'interprétation.

On suppose que l'analyse de plusieurs documents est nécessaire pour reconstruire la structure d'un discours, ce dernier pouvant finalement être assemblé comme une "mosaïque » (Keller 2011, p. 62). L'analyse réalisée dans l'article s'appuie sur 144 prises de position, autour de la problématique de la perméabilité, des principaux acteurs politiques des deux domaines organisationnels de l'enseignement supérieur et de la formation professionnelle, en Allemagne. Les documents de synthèse comportaient des déclarations de politique éducative, des communiqués de presse, des discours protocolaires et des contributions à des commissions parlementaires.

Selon la différenciation de Schmidt (2008), les acteurs sociaux ont été sélectionnés comme faisant partie de l'espace de discours coordonné. L'analyse se concentre donc sur les acteurs qui sont au centre de la formulation des politiques (voir tableau 1 ci-après). 
Tableau 1. Documents examinés pour l'analyse du discours

\begin{tabular}{|c|c|c|c|}
\hline \multirow[t]{2}{*}{ Les représentants de/des } & \multirow[t]{2}{*}{ Acteurs sélectionnés } & $\begin{array}{l}1990- \\
1998\end{array}$ & $\begin{array}{l}1999- \\
2012\end{array}$ \\
\hline & & N & N \\
\hline $\begin{array}{l}\text { Gouvernement national (ministères } \\
\text { de l'Éducation) }\end{array}$ & $\begin{array}{l}\text { Bundesministerium für Bildung und Forschung (BMBF) } \\
\text { [Ministère fédéral de l'Éducation et de la Recherche]. }\end{array}$ & 11 & 22 \\
\hline États fédéraux & $\begin{array}{c}\text { Kultusministerkonferenz (KMK) } \\
\text { [Conférence permanente des ministres de l'Éducation et des Affaires } \\
\text { culturelles des Länder]. }\end{array}$ & 5 & 24 \\
\hline $\begin{array}{l}\text { Organismes d'enseignement } \\
\text { supérieur }\end{array}$ & $\begin{array}{l}\text { Hochschulrektorenkonferenz (HRK) } \\
\text { [Conférence des Recteurs Allemands]. }\end{array}$ & 8 & 14 \\
\hline Entreprises/employeurs & $\begin{array}{l}\text { Deutscher Industrie- und Handelskammertag (DIHK) [Association des } \\
\text { chambres de commerce et d'industrie allemandes]. }\end{array}$ & 4 & 18 \\
\hline Syndicats & $\begin{array}{l}\text { Deutscher Gewerkschaftsbund (DGB) } \\
\text { [Confédération des syndicats allemands] }\end{array}$ & 8 & 22 \\
\hline Co-auteurs & & & 8 \\
\hline $\mathrm{N}$ & & 36 & 108 \\
\hline
\end{tabular}

Source : Bernhard (2017a, p. 125).

Le choix des documents repose sur la conception de la perméabilité comme concept de sensibilisation. Dans un premier temps, tous les documents du discours politique qui se concentrent sur les structures reliant largement l'enseignement supérieur et l'EFP ont été choisis pour dégager une vision d'ensemble du discours éducatif. Dans un deuxième temps, seuls les documents abordant la question de la perméabilité ont été analysés. Comme le suggère Keller (2011), la sélection des documents et l'analyse reconstructive séquentielle sont fondées sur la méthodologie de la théorie enracinée, de Strauss \& Corbin (1996), qui utilise des stratégies d'échantillonnage théorique, de codage contrasté, ouvert, axial et sélectif. Selon Keller (2011), l'analyse des discours peut se concentrer sur différents éléments ; dans cet article, l'accent est mis sur des parties de la structure dite phénoménale, qui se compose des éléments nécessaires pour qu'un sujet soit construit comme un problème dans l'agenda public.

Ces éléments apportent des réponses aux questions suivantes : quel est le problème ? Pourquoi est-ce un problème ? Que faut-il faire pour le résoudre ? Qui devrait le faire ? Qui devrait en bénéficier? Adaptées à cet article, les questions sont les suivantes : quel est le problème global de perméabilité et comment la perméabilité est-elle conceptualisée ? Au cas où la question des structures d'appui serait pertinente dans les documents, les questions suivantes sont intéressantes : pourquoi les structures de soutien sont-elles nécessaires ? Quelles sont les structures nécessaires ? pour qui, et qui est responsable?

La dimension réglementaire a été examinée principalement à travers l'analyse de la littérature secondaire, mais aussi à l'aune de l'analyse de documents juridiques basés sur le concept de perméabilité. II s'agit des lois sur l'enseignement supérieur des Länder, de la loi sur la formation professionnelle (BBIG), de la loi fédérale sur l'enseignement supérieur et des décisions de la KMK (Kultusministerkonferenz).

$\left(^{*}\right)$ : Pour une description détaillée de la procédure d'analyse et d'échantillonnage des documents, ainsi que pour consulter la liste des documents examinés, voir Bernhard (2017a, b).

$(* *)$ : Il s'agit de l'analyse de discours du point de vue de la sociologie de la connaissance. 


\section{Les besoins hétérogènes des diplômés de l'enseignement professionnel : un sujet en évolution}

Dans cette partie, dans un premier temps, nous présenterons les principaux développements discursifs et régulateurs relatifs à la perméabilité entre l'enseignement supérieur et la formation professionnelle. Nous examinerons, ensuite, l'évolution des structures d'appui visant à satisfaire les besoins hétérogènes des personnes diplômées de l'enseignement professionnel. Enfin, nous évoquerons les réflexions autour des structures d'information et de conseil, l'organisation des études, la pédagogie et le financement.

\subsection{Les discours élargis sur la perméabilité}

En Allemagne, la question de la perméabilité est évidente. Avant 1999, la perméabilité était principalement discutée sous l'angle de l'amélioration de l'accès à l'enseignement supérieur. Dans une certaine mesure, le discours portait également sur l'établissement de liens organisationnels sous la forme de programmes d'études en alternance, reconnus comme programmes officiels d'enseignement supérieur, en 1995. Au cours de cette première période, l'objectif n'était cependant pas d'augmenter le nombre d'étudiants, mais de renforcer l'attractivité de la formation professionnelle initiale en raison d'une pénurie de travailleurs qualifiés. L'institutionnalisation de possibilités de formation continue dans l'enseignement supérieur a été considérée comme l'un des moyens d'atteindre cet objectif. L'extrait suivant révèle la préoccupation principale :

"Le problème que nous avons avec l'équivalence, qui est aussi le point de départ des choix éducatifs, c'est que de nombreux parents et de nombreux jeunes considèrent la formation professionnelle comme une impasse, une voie à sens unique qui, une fois empruntée, ne peut être abandonnée facilement. Il est donc important - et c'est également notre objectif-de rendre la formation professionnelle et les perspectives de carrière suffisamment attrayantes pour que la concurrence entre les différentes filières d'études puisse effectivement s'exercer. " (BMBW, 1992, p. 69$)^{3}$

Après 1999, le concept étroit de perméabilité, tel que véhiculé par le discours allemand sur la politique de l'éducation, s'est transformé en un concept multidimensionnel, englobant la question de l'accès à l'éducation, ainsi que la notion de reconnaissance et de validation des compétences professionnelles. Cela signifie que l'équivalence des compétences acquises dans l'EFP et l'enseignement supérieur a été reconnue et socialement construite pour la première fois. Les liens organisationnels, matérialisés par les programmes d'études en alternance, ont également été considérés comme un facteur d'augmentation de la perméabilité. Enfin, avec l'arrivée d'un nouveau groupe d'étudiants, les structures d'études existantes, qui se concentraient uniquement sur les besoins des étudiants traditionnels, ont été remises

3. L'auteur a traduit toutes les citations directes présentes dans cet article. 
en question. Les modifications des structures ont été de plus en plus discutées afin de mieux répondre aux besoins différenciés de la population étudiante.

Cette montée en puissance de la gestion de l'hétérogénéité est liée, en particulier, au lancement du programme de politique éducative "Aufstieg durch Bildung : offene Hochschulen ", en 2008. En outre, de nouvelles réglementations concernant l'accès à l'enseignement supérieur des personnes disposant de qualifications professionnelles ont été introduites avec la décision de la KMK, en 2009. Ces nouvelles réglementations peuvent s'apparenter à un changement de paradigme car, pour la première fois en Allemagne, les individus peuvent bénéficier d'un droit à l'enseignement supérieur par le biais de la filière de formation professionnelle.

Toutefois, seuls les titulaires d'un diplôme sanctionnant la fin d'un enseignement professionnel avancé (ISCED 5B) peuvent y prétendre. Les titulaires d'un diplôme initial de l'EFP doivent acquérir au moins trois ans d'expérience professionnelle. En outre, souvent ils doivent réussir un test d'entrée pour accéder à des formations de l'enseignement supérieur en lien avec leur qualification professionnelle (KMK, 2009).

Dans ce contexte de changement structurel des conditions d'accès, il est intéressant d'analyser dans quelle mesure cela modifie également, sur le plan institutionnel, les structures d'appui à ce nouveau groupe d'étudiants. L'aide aux étudiants disposant de qualifications professionnelles ayant été peu abordée avant 1999, seuls les débats après 1998 feront l'objet d'une analyse plus approfondie.

\subsection{Des modifications réglementaires mineures des structures de soutien}

En Allemagne, le cadre réglementant les structures d'appui pour les étudiants diplômés de l'enseignement professionnel n'a pas connu de modifications significatives. Dans de nombreux cas, des changements ont été initiés et financés par le biais d'initiatives résultant de programmes politiques, sans pour autant faire l'objet de nouvelles obligations légales pour les organisations d'enseignement supérieur. Par exemple, en 2010, le concours «Aufstieg durch Bildung : offene Hochschulen " a été approuvé par le gouvernement fédéral et les Länder. Il vise à développer, au sein des établissements d'enseignement supérieur, des services innovants de formation tout au long de la vie, axés sur la demande et ancrés dans la durée (Banscherus \& al., 2016). Pourtant, nul ne peut dire dans quelle mesure les programmes se poursuivront lorsque le financement du concours sera épuisé.

Outre ces programmes novateurs, les lois sur l'enseignement supérieur des Länder ont renforcé l'introduction de programmes d'études à temps partiel et, dans certains cas, ont explicitement promu de meilleures conditions d'études pour les étudiants qualifiés et actifs (Bernhard, 2017a). Parallèlement, grâce à l'instauration, en 2008, d'une bourse d'études

4. En français : «le progrès par l'éducation : des universités ouvertes ». 
attribuée par le gouvernement, appelée Aufstiegsstipendium5 ${ }^{5}$ des possibilités de financement pour les étudiants diplômés de l'enseignement professionnel particulièrement performants ont été introduites.

\subsection{Soutenir les étudiants diplômés d'EFP : motifs universalistes et fonctionnalistes}

En Allemagne, la question de la perméabilité entre l'enseignement supérieur et la formation professionnelle a donné lieu à de vifs débats. Deux discours contradictoires ont pu être identifiés : un discours structurel conservateur qui rejette l'idée d'une plus grande perméabilité entre l'enseignement supérieur et la formation professionnelle et un discours structurel critique qui, au contraire, promeut une perméabilité accrue. Le discours structurel-conservateur a peu abordé la question des structures de soutien, et en 2009, lorsque l'accès à l'enseignement a été libéralisé, cette question est devenue peu pertinente. Dans le droit fil de l'argumentation structurelle-conservatrice, le soutien aux étudiants diplômés de l'enseignement professionnel est justifié par la nécessité d'assurer la qualité de l'enseignement supérieur, puisque ces étudiants ne sont pas aussi bien préparés pour étudier que les étudiants traditionnels :

"L'expérience a également montré qu'il y a certainement des difficultés d'intégration. Typiquement, lorsqu'il s'agit d'une matière comme les mathématiques et les sciences naturelles ou l'anglais, des connaissances de base sont exigées. Nous n'avons pas du tout besoin de parler d'autres langues étrangères. C'est une raison, pour les universités, de soutenir ces étudiants, soit pendant la phase d'intégration, soit pour leur offrir des cours préparatoires si elles [les universités] en ont les moyens. "(HRK, 2010)

Dans le discours structurel-critique, une argumentation universaliste et fonctionnaliste pourrait être reconstruite. D'un point de vue universaliste, les structures d'appui sont essentielles pour traduire l'idéal du droit à l'éducation et réduire les inégalités. D'un point de vue fonctionnaliste, ces structures servent avant tout à accroître l'attrait de l'enseignement supérieur auprès de nouveaux groupes d'étudiants, les universités devenant de plus en plus dépendantes de ces derniers en raison des changements démographiques :

"Les universités doivent d'abord se rendre compte qu'à un moment donné, elles auront aussi besoin des diplômés de la formation professionnelle comme clients. Elles n'ont pas encore ce besoin [...]. Mais si la tendance démographique s'accentue vraiment, et il ne s'agit pas uniquement de leur donner un accès, d'une manière ou d'une autre, mais aussi de développer des cursus d'études sur mesure. " (DIHK, 2010, p. 43)

5. En français : «bourses d'avancement ». 
En même temps, les structures de soutien devraient permettre d'accroître l'efficacité des études et de diminuer les changements d'études coûteux, ainsi que les abandons. L'analyse du discours a révélé que ces deux postures étaient présentes dans les prises de position de presque tous les acteurs. Cela signifie qu'un acteur peut critiquer la perméabilité tout en reconnaissant sa nécessité d'un point de vue fonctionnel. Néanmoins, certains acteurs, comme les représentants de l'enseignement général et supérieur (KMK et HRK), se sont positionnés plus souvent davantage du côté du discours structurel conservateur et en faveur des privilèges dont jouit l'enseignement général dans la société. Par ailleurs, les syndicats ont formulé des critiques structurelles plus universalistes et les employeurs ont plaidé en faveur d'une plus grande perméabilité, à condition qu'elle soit efficace et pertinente pour le marché du travail (Bernhard, 2017a).

\subsection{Comment soutenir les étudiants diplômés de l'enseignement professionnel?}

Les différentes tendances du discours allemand en matière d'information et de conseil, de structures d'études, de pédagogie et de soutien financier sont évoquées ci-après.

\subsubsection{Services d'information et de conseil : améliorer l'adéquation et augmenter l'intérêt pour la poursuite d'études}

Selon le discours critique structurel fonctionnaliste allemand, des conseils et des informations sont nécessaires pour favoriser une meilleure adéquation entre les étudiants et les organisations de l'enseignement supérieur. Les procédures d'auto-évaluation devraient permettre d'informer les étudiants titulaires de diplômes de l'EFP sur les défis qui les attendent, afin qu'ils puissent prendre une décision éclairée (DIHK \& HRK, 2008). Cependant, aucun type d'organisation de l'enseignement supérieur n'est conçu spécifiquement pour les personnes diplômées de l'enseignement professionnel. Elles sont donc libres d'intégrer les universités, les hautes écoles spécialisées ou d'autres types d'établissements.

En Allemagne, selon les arguments universalistes, les structures de conseil et d'information devraient permettre d'expliciter les possibilités d'études, de renforcer l'intérêt de la poursuite d'études pour les personnes diplômées de l'enseignement professionnel et de les conseiller sur la manière dont leurs souhaits peuvent être réalisés. Le but de l'information et du conseil est de motiver ces personnes à intégrer un programme d'études.

\subsubsection{Des structures d'études flexibles, mais sans pédagogie adaptée}

En Allemagne, le groupe des diplômés professionnels est construit, dans les discours, comme un groupe distinct - au regard de diverses caractéristiques - des étu- 
diants dits traditionnels qui entrent à l'université directement après avoir obtenu leur diplôme d'études générales secondaires. Le futur étudiant diplômé de l'EFP apparaît alors comme quelqu'un qui a réussi dans sa profession et qui a suivi une formation continue. Comme ces personnes sont habituellement plus âgées, elles ont souvent des obligations familiales et ne peuvent renoncer à un revenu au profit d'un programme d'études à temps plein :

"Aucune personne, dès lors qu'elle a 28 ans, qu'elle travaille, qu'elle a déjà un diplôme de Meister, qu'elle a fondé une famille, n'abandonnera tout pour obtenir par la suite un diplôme universitaire. " (DIHK \& HRK, 2008)

Par conséquent, les dispositifs permettant à ces étudiants de continuer à travailler tout en remplissant leurs obligations familiales, comme les cours à temps partiel ou à distance et les cours du soir, sont considérés comme particulièrement opportuns et ont été réclamés de façon récurrente dans le cadre du discours structurel critique.

Lorsqu'il s'agit de la conception du contenu de l'étude ou de la pédagogie, les positions divergent dans les discours. Selon le discours structurel-critique fonctionnaliste, mais aussi selon le discours structurel conservateur, des cours préparatoires sont nécessaires pour les futurs étudiants (HRK 2008). Ainsi, la réussite des études peut être facilitée (structurel-critique fonctionnaliste), et la qualité de l'enseignement supérieur assurée (structurel conservateur) :

"En même temps, la HRK et la DIHK recommandent aux universités d'élargir autant que possible les cours préparatoires et de transition afin de combler les lacunes des personnes ayant des qualifications professionnelles, surtout s'agissant des travaux et contenus scientifiques qui ne sont pas couverts par la matière concernée. " (DIHK \& HRK, 2008)

Ainsi, les personnes diplômées de l'enseignement professionnel sont considérées comme un groupe à risque, ce qui nécessite une adaptation particulière aux exigences requises par la poursuite d'études. Il s'agit donc d'une adaptation unilatérale des diplômés de l'enseignement professionnel aux exigences des universités. L'adaptation de ces dernières et la manière dont elles intègrent ces étudiants en leur sein, tant en ce qui concerne la prise en compte de leurs compétences que de leurs expériences, sont au coeur du discours universaliste :

"Mais je pense aussi, par exemple, à développer des méthodes d'enseignement flexibles qui tiennent compte des stratégies acquises individuellement et des particularités des différents groupes d'apprentissage et groupes cibles. " (KMK, 2011, p. 9)

Dans le cadre de ce discours universaliste, des cours préparatoires sont également considérés comme utiles. Toutefois, ces cours ne s'adressent pas uniquement aux diplômés de l'EFP, mais à tous ceux qui en ont besoin. 


\subsubsection{Un soutien financier pour tous ou seulement pour les plus performants?}

Selon le discours universaliste allemand, les exigences en matière de structures de soutien financier prévalent. D'une part, les structures existantes de soutien financier individuel, telles que prévues par la $B A f o ̈ G^{6}$, devraient s'adapter au nouveau groupe d'étudiants en supprimant les restrictions d'âge. Deuxièmement, on devrait attribuer davantage de fonds, destinés aux titulaires de diplômes professionnels, aux organismes d'enseignement supérieur. Cela inciterait à reconnaître la place de ces diplômés et à les considérer comme un groupe pertinent d'étudiants (DGB, 2011). Le discours fonctionnaliste plaide pour l'attribution de bourses visant à attirer uniquement des personnes particulièrement talentueuses possédant des qualifications acquises au sein de l'EFP. L'introduction de l'Aufstiegsstipendium ${ }^{7}$, une bourse créée en 2008 en Allemagne, répond à cette attente.

\section{Conclusion : une perméabilité accrue grâce à des structures de soutien sélectionnées et des hiérarchies persistantes}

Cet article a montré qu'en Allemagne, les structures institutionnelles favorisant une plus grande perméabilité entre l'enseignement supérieur et l'enseignement et la formation professionnels se sont développées au fil du temps. Avec la reconnaissance des étudiants diplômés de l'enseignement professionnel comme étudiants légitimes, dans les années 2000, les questions relatives à la manière de traiter ce groupe d'étudiants au sein des organisations d'enseignement supérieur sont devenues de plus en plus prégnantes. En Allemagne, le discours structurel critique visant à accroître la perméabilité gagne du terrain, même si le discours structurel conservateur demeure. Par conséquent, si d'une part, la perméabilité est exigée, les hiérarchies existantes entre les parcours éducatifs généraux et les parcours professionnels sont, dans le même temps, reproduites.

C'est le cas lorsque les étudiants titulaires de diplômes professionnels sont surtout considérés, dans les discours, comme un groupe ayant des lacunes qu'il convient de combler. Ainsi, dans le même temps, on assiste à une homogénéisation des étudiants de l'EFP, tandis que les différences et l'absence d'équivalence entre les "étudiants traditionnels » et les étudiants diplômés d'EFP sont reproduites, en particulier dans le

6. BAföG est l'acronyme de Bundesausbildungsförderungsgesetz (loi fédérale allemande sur l'aide à la formation). Grâce à ce programme, les étudiants bénéficient d'un soutien pour couvrir leurs frais de subsistance et de formation afin de réduire l'obligation de travailler pendant leurs études. Le niveau de soutien financier dépend des revenus et des biens de l'étudiant, ainsi que des revenus de ses parents. La moitié de l'aide aux étudiants de l'enseignement supérieur prend la forme d'une bourse, l'autre moitié celle d'un prêt public sans intérêt.

7. En français : «bourses d'avancement». 
discours structurel conservateur. Cependant, les recherches actuelles montrent qu'en Allemagne, aucune différence significative dans les résultats des études ne peut être mise en évidence entre les groupes d'étudiants titulaires de diplômes d'EFP et d'autres étudiants (Wolter \& al., 2017).

L'absence d'équivalence est aussi visible dans le fait qu'une élite très performante de diplômés professionnels - caractérisée par de nombreuses années d'apprentissage et une expérience professionnelle plus riche qu'un diplômé de l'enseignement général titulaire de l'Abitur - est désignée comme un groupe cible, contrairement aux diplômés " moyens " de l'EFP. Cependant, dans les débats, il n'y a pas de restriction relative à l'accès aux établissements pour ces étudiants. En Allemagne, les étudiants diplômés de l'enseignement professionnel sont également décrits comme se distinguant par leur âge, leur statut professionnel et leurs responsabilités familiales. Les questions de financement et d'organisation des études sont donc essentielles.

En résumé, même si les discours contradictoires réclament la mise en place de nombreuses formes similaires de structures de soutien, on observe une grande différence concernant le fait de savoir si les personnes titulaires de diplômes professionnels constituent un groupe cible désiré ou imposé. En outre, il apparaît clairement que les structures d'appui peuvent être conçues, soit pour différencier les étudiants diplômés d'EFP des étudiants " traditionnels " et reproduire ainsi une différence entre eux, soit pour les inclure. Ce dernier aspect ressort principalement du discours universaliste.

Cependant, comme l'accent mis sur les différences dominait dans les discours, l'absence d'équivalence et la reproduction des hiérarchies perdurent. Il conviendrait à l'avenir de déterminer quel discours devient dominant et comment les structures sont mises en place et vécues au sein des organisations. Dans l'ensemble, les diplômés de l'EFP sont aujourd'hui reconnus comme un nouveau groupe d'étudiants pertinent et les structures de soutien sont considérées comme nécessaires. Cela peut représenter une amélioration majeure par rapport à une époque où ce groupe n'était même pas considéré comme légitime. 


\section{Encadré 2. Documents cités du corpus d'analyse $\left({ }^{*}\right)$}

BMBW [Bundesministerium für Bildung und Wissenschaft] (1992). Gleichwertigkeit beruflicher und allgemeiner Bildung. Rede von Fritz Schaumann. 1. BMBW-Fachtagung am 8.07.1992. Bonn: Bundesministerium für Bildung und Wissenschaft.

DGB [Deutscher Gewerkschaftsbund] (2011). Deutschland ist weit von einer Bildungsrepublik entfernt. Pressemitteilung vom 13.09.2011

DIHK [Deutscher Industrie- und Handelskammertag] (2010). Öffentliche Anhörung zum Thema "Europäischer Qualifikationsrahmen/Deutscher Qualifikationsrahmen". Redebeiträge von Sybille von Obernitz.: In: Deutscher Bundestag: Protokoll 17/19 des Ausschusses für Bildung, Forschung und Technikfolgenabschätzung. Berlin.

DIHK \& HRK (2008). Für mehr Durchlässigkeit zwischen beruflicher Bildung und Hochschulbildung! Gemeinsame Erklärung des DIHK und der HRK.

HRK [Hochschulrektorenkonferenz] (2008). Neuordnung des Hochschulzugangs für beruflich Qualifizierte. Entschließung der Mitgliederversammlung am 18.11.2008. HRK. Bonn.

HRK (2010). Öffentliche Anhörung zum Thema „Europäischer Qualifikationsrahmen/ Deutscher Qualifikationsrahmen“. Redebeiträge von Jan Rathjen. In: Deutscher Bundestag: Protokoll 17/19 des Ausschusses für Bildung, Forschung und Technikfolgenabschätzung. Berlin.

KMK [Kultusministerkonferenz] (2011). Grußwort der Kultusministerkonferenz. Rede von Prof. Dr. Birgitta Wolff. Auftaktveranstaltung "Wettbewerb Aufstieg durch Bildung: offene Hochschulen" am 9.12.2011.

$\left(^{*}\right)$ : Une liste de tous les documents analysés est disponible dans Bernhard (2017b) https:// shop.budrich-academic.de/wp-content/uploads/2015/10/Bernhard_Onlineanhang. pdf?v=3a52f3c22ed6 [02.11.2018]

\section{Bibliographie}

Baethge M. (2006), "Das deutsche Bildungs-Schisma: Welche Probleme ein vorindustrielles Bildungssystem in einer nachindustriellen Gesellschaft hat SOFI-Mitteilungen", $\mathrm{n}^{\circ} 34$ Soziologisches Forschungsinstitut an der Universität Göttingen, pp. 13-27.

Baethge M. \& Wolter A. (2015), “The German skill formation model in transition: from dual system of VET to higher education?", Journal for Labour Market Research, 48(2), pp. 97-112.

Banscherus U., Bernhard N., Graf L. (2016), Durchlässigkeit als mehrdimensionale Aufgabe. Bedingungen für flexible Bildungsübergänge, Berlin: Friedrich-Ebert-Stiftung.

Bernhard N. (2017a), Durch Europäisierung zu mehr Durchlässigkeit? Veränderungsdynamiken des Verhältnisses von Berufs-und Hochschulbildung in Deutschland und Frankreich. Opladen, Berlin, Toronto Budrich UniPress Ltd.

Bernhard N. (2017b), Online-Anhang zum Buch: Durch Europäisierung zu mehr Durchlässigkeit? Veränderungsdynamiken des Verhältnisses von Berufs- und 
Hochschulbildung in Deutschland und Frankreich. https://shop.budrich-academic. de/wp-content/uploads/2015/10/Bernhard_Onlineanhang.pdf?v=3a52f3c22ed6 [02.11.2018]

Busemeyer M. R. \& Trampusch C. (Eds.) (2012), The political economy of collective skill formation. Oxford University Press.

Euler D. (2013), Das duale System in Deutschland - Vorbild für einen Transfer ins Ausland? Eine Studie im Auftrag der Bertelsmann Stiftung, https:/www.bibb.de/dokumente/ pdf/xcms_bst_dms_37640_37641_2.pdf [15.05.2018]

Graf L. (2013), he Hybridization of Vocational Training and Higher Education in Austria, Germany and Switzerland. Opladen: Budrich UniPress.

Hanft A., Zawacki-Richter, O. \& Gierke W. B. (Eds.) (2015), Herausforderung Heterogenität beim Übergang in die Hochschule. Münster: Waxmann.

Keller R. (2006), "Wissenssoziologische Diskursanalyse”, in Keller R., Hirseland A., Schneider W., \& Viehöver W. (Eds.), Handbuch Sozialwissenschaftliche Diskursanalyse. Band 1: Theorien und Methoden Opladen: Leske+Budrich, pp. 115-146.

Keller R. (2011), "The Sociology of Knowledge Approach to Discourse (SKAD)”, Human Studies, 34(1), pp. 43-65.

Kerckhoff A. C. (2001), "Education and Social Stratification Processes in Comparative Perspective", Sociology of Education, 74 (Extra Issue), pp. 3-18.

KMK (2009), Hochschulzugang für beruflich qualifizierte Bewerber ohne schulische Hochschulzugangsberechtigung. Beschluss der Kultusministerkonferenz vom 06.03.2009.

Pollak R. \& Reimer D. (2010), "Educational Expansion and Its Consequences for Vertical and Horizontal Inequalities in Access to Higher Education in West Germany", European Sociological Review, 26(4), pp. 415-430.

Powell J. J. W, Graf L. ; Bernhard N., Coutrot L. ; Kieffer A. (2012), “The Shifting Relationship between Vocational and Higher Education in France and Germany: Towards Convergence?”, European Journal of Education 47(3), pp. 405-423.

Powell J. J. W. \& Solga H. (2011), "Why are Participation Rates in Higher Education in Germany so Low? Institutional Barriers to Higher Education Expansion”, Journal of Education and Work, 24(1-2), pp. 49-68.

Schmidt V. A. (2008), "Discursive Institutionalism: The Explanatory Power of Ideas and Discourse", Annual Review of Political Science, 11, pp. 303-326.

Scott W. R. (2008), Institutions and Organizations: Ideas and Interests (3rd Edition), Thousand Oaks: Sage. 
Shavit Y. \& Müller W. (2000), "Vocational Secondary Education: Where Diversion and Where Safety Net?", European Societies, 2(1), pp. 29-50.

Strauss A. \& Corbin J. (1996), Grounded Theory: Grundlagen Qualitativer Sozialforschung, Weinheim: Beltz, Psychologie Verlags Union.

Ulbricht L. (2012), “Öffnen die Länder ihre Hochschulen? Annahmen über den Dritten Bildungsweg auf dem Prüfstand”, Die Hochschule, 21(1), pp. 154-168.

Wolter A., Banscherus U., Kamm C., Otto A. \& Spexard A. (2014), "Durchlässigkeit zwischen beruflicher und akademischer Bildung als mehrstufiges Konzept. Bilanz und Perspektiven”, Beiträge zur Hochschulforschung, 36(4), pp. 8-39.

Wolter A., Dahm G., Kamm C., Kerst C. \& Otto A. (2017), Nicht-traditionelle Studierende: Studienverlauf, Studienerfolg und Lernumwelten, Berlin und Hannover.

Young M., Spours K., Howieson C. \& Raffe D. (1997), "Unifying academic and vocational learning and the idea of a learning society", Journal of Education Policy, 12(6), pp. 527-537. 